\title{
IMPORTANCE DES PROTÉINES DU LIQUIDE CELOMIQUE SUR LA FERTILITÉ DES GAMĖTES CHEZ LA TRUITE ARC-EN-CIEL ET POSSIBILITÉ DE SUBSTITUTION.
}

\author{
R. BILLARD \\ Laboratoire de Physiologie des Poissons \\ I.N.R.A., 78350 JOUY EN JOSAS
}

\section{RÉSUMÉ}

Des ovules de truite arc-en-ciel ont été inséminés avec des spermatozoïdes ayant reçu un lavage dans un milieu voisin de la composition minérale du liquide séminal (MMLS) et dans lequel les spermatozoïdes demeurent immobiles. Le lavage diminue significativement $(P<0,01)$ le pouvoir fécondant et, lorsque l'insémination est pratiquée dans une solution de $\mathrm{NaCl}$ (DI), la chute de fertilité est plus forte que dans le liquide cœlomique.

Le lavage des ovules dans un milieu minéral de liquide cœlomique (MMLC) ou dans le DIA provoque une diminution de la fécondabilité $(P<0,01)$ qui est compensée par une augmentation de la quantité de spermatozoïdes apportés lors de l'insémination. Le lavage combiné des spermatozoïdes et des ovules provoque une diminution accrue du taux de fécondation.

Des essais de complémentation des dilueurs minéraux avec des protéines (BSA et ovalbumine) ont montré que la BSA prolongeait la durée de motilité des spermatozoïdes après dilution dans DI et cet effet est interprété comme résultant d'une action protectrice de la BSA contre l'effet de dilution. Cependant le taux de fécondation n'est pas amélioré par l'addition de BSA au dilueur d'insémination. L'effet de l'ovalbumine s'est toujours révélé défavorable. Si l'incorporation de protéines comme la BSA au dilueur d'insémination ne se justifie pas pour la pratique courante de l'insémination artificielle, elle reste envisageable dans le cas de milieux destinés à des manipulations de gamètes comme leur conversation ou leur transport.

\section{Importance of colomic fluid protein in rainbow trout gamete fertilization and} its possible substitution.

\section{SUMMARY}

Rainbow trout ova were inseminated with spermatozoa washed in a medium (MMLS) having a mineral composition similar to seminal fluid and in which the spermatozoa were motionless. Washing significantly decreased $(P<0,01)$ fertilizing ability, but when insemination was done in a $\mathrm{NaCl}$ solution (DI), fertility was much lower than when it was done in cœlomic fluid.

Washing ova in a mineral cœlomic fluid medium (MMLC) or in DIA caused the fertility rate to drop (P<0.01); this was compensated for by an increase in the number of spermatozoa supplied during insemination. Washing spermatozoa and ova together caused the fertilization rate to drop even more.

Trials complementing mineral diluents with proteins (BSA and ovalbumin) showed that BSA always prolonged spermotozoon motility after DI dilution and, this effect was interpreted as resulting from a protective BSA effect against the dilution effect. However, adding $B S A$ to the insemination diluent did not improve the fertilization rate. Ovalbumin always had an unfavorable influence. While incorporating proteins such as BSA into the insemination diluent is not justified in the current practise of artificial insemination, it may be used for media in which gametes are manipulated for storage or transport.

\section{INTRODUCTION}

Dans la méthode d'insémination dite sèche comme dans celle faisant appel à un dilueur (BILLARD et al., 1974 a), le liquide cœlomique est éliminé du fait des variations de volume et de composition, la présence de liquide d'hydropsie (DORIER, 1949) et aussi du 
fait qu'il contient tout le vitellus des ovules ayant pu se trouver écrasés lors des opérations de prélèvement. Ce vitellus qui précipite au contact de l'eau "piège " les spermatozoïdes et réduit fortement le taux de fécondation (LEITRITZ et LEWIS, 1976; BILLARD, 1977 a). Afin de rendre plus complète l'élimination de ces substances indésirables, NOMURA a développé au Japon une technique qui comporte le lavage des ovules avec une solution physiologique avant insémination (BILLARD, 1977 b). Nous avons proposé de notre côté une technique d'insémination avec emploi d'un dilueur qui vient se substituer en quantité bien définie au liquide cœlomique (BILLARD, 1975 a). On utilise ainsi un milieu de qualité constante et dont la quantité est facilement contrôlable; par souci de n'employer que le nombre optimum de spermatozoïdes on applique des taux de dilution relativement élevés $(1 / 100$ à 1/1000). Cela est lié à la grande taille de l'ovule et à la nécessité de respecter un rapport numérique bien défini.entre quantité de dilueur et de gamètes (BILLARD, 1975 a).

On sait que le liquide cœlomique contient des substances susceptibles d'interagir avec les spermatozoïdes (gamones de HARTMAN, 1944 ; voir MANN 1956 et GINSBURG, 1968 pour revue) et de stimuler leur motilité (YOSHIDA et NOMURA, 1972).

On est donc conduit à se demander si l'élimination du liquide ccelomique ne présente pas quelques inconvénients en regard des avantages susmentionnés qu'il présente par ailleurs.

Dans le présent travail, l'étude de l'influence du liquide cœlomique et aussi du liquide séminal est abordée par des expériences de lavage des gamètes. En outre, dans le but de trouver un substitut au liquide cœlomique on a procédé à l'incorporation de protéines dans les dilueurs de conservation ou d'insémination et testé leurs effets sur la survie des gamètes.

\section{MATÉRIEL ET MÉTHODES}

Des géniteurs adultes âgés de 4-5 ans en provenance de la pisciculture de la Bigottière sont entrés au Laboratoire au cours de la période de reproduction. Les expériences se déroulent en janvier, c'est-à-dire deux mois environ après le début de la spermiation. Les femelles sont utilisées dans les quelques jours qui suivent l'ovulation. L'expérimentation fait appel à un schéma antérieurement décrit (BILLARD et al., 1974 a) avec testage des effets des traitements des gamètes et des potentialités des dilueurs sur des lots d'environ 200 ovules placés dans $10 \mathrm{ml}$.de dilueur, inséminés avec 1 ou $10 \mu \mathrm{l}$ de sperme (dilution $1 / 10.000$ et 1/1000) et incubés séparément à température constante $10 \pm 10 \mathrm{C}$. Les effets sont en définitive appréciés par le pourcentage d'cuts embryonnés établi à 150 degrés-jours.

Les expériences suivantes ont été réalisées:

\section{Expérience 1 : Lavage des spermatozoïdes}

Des lots d'ovules recevant comme dilueur $10 \mathrm{ml}$ de liquide cœlomique ou $10 \mathrm{ml}$ d'un dilueur d'insémination (DI) (solution de $\mathrm{NaCl}$ po $=250$ mosmoles, $\mathrm{pH}=9.0$, tampon de carbonate bicarbonate $=0.02 \mathrm{M}$ ) sont inséminés soit avec du sperme complet (témoin) ou des spermatozoïdes lavés dans les conditions suivantes : après centrifugation pendant $30 \mathrm{mn}$ à $1800 \mathrm{~g}$ et $4^{\circ} \mathrm{C}$ (selon NOMURA, 1964), le liquide séminal est éliminé et le culot de spermatozoïdes resuspendu dans 10 volumes d'un milieu minéral voisin de la composition du liquide séminal: MMLS (BILLARD et JALABERT, 1974). L'ensemble est agité pendant une minute puis centrifugé comme ci-dessus, le surnageant est éliminé et du MMLS est ajouté en quantité équivalente au liquide séminal dans le sperme témoin afin de reconstituer le spermatocrite initial. On sait que le MMLS par sa haute teneur en $\mathrm{K}^{+}$maintient les spermatozoïdes à l'état immobile de sorte que les spermatozoïdes $n$ 'ont pas été mis en mouvement lors des opérations de lavage (BILLARD, 1975 b).

Expérience 2: Lavage des ovules suivants:

Du sperme frais a été utilisé pour inséminer des ovules qui ont subi les traitements

- ovules témoins non lavés simplement séparés du liquide cœlomique lors du prélèvement.

- ovules lavés: des lots d'environ 200 ovules sont lavés dans $50 \mathrm{ml}$ des milieux suivants :

- liquide cœlomique naturel recueilli lors du prélèvement des ovules ;

- liquide colomique reconstitué quant à ses composants minéraux (MMLC, 250 mosm., pH 9.0, BILLARD et JALABERT, 1974). 
- dilueur d'insémination à 250 mosm. (DI).

- eau douce.

Après légère agitation pendant une minute, le milieu de lavage est éliminé par égouttage et remplacé par $10 \mathrm{ml}$ de dilueur d'insémination (DI) dans lequel 1 ou $10 \mu \mathrm{l}$ de sperme sont ajoutés (dilution $1 / 10.000$ et $1 / 1000$ ).

Expérience 3 : Lavage combiné des ovules et des spermatozoïdes.

L'insémination est pratiquée dans le DI avec des spermatozoïdes et ovules lavés, des spermatozoïdes dans les mêmes conditions que l'expérience 1 et des ovulespendant 1 minute dans MMLC et DI. L'expérimentation a été faite en double.

Expérience 4 : Essais d'incorporation de protéines dans les dilueurs.

Des essais de reconstitution au moins partielle de liquide cœlomique par incorporation d'une composante protéique ont porté sur deux milieux, l'un destiné à la conservation du sperme à l'état immobile (MMLS) et l'autre (DI) destiné à l'insémination, au stockage des ovules et à tester la durée de survie des spermatozoïdes à l'état motile.

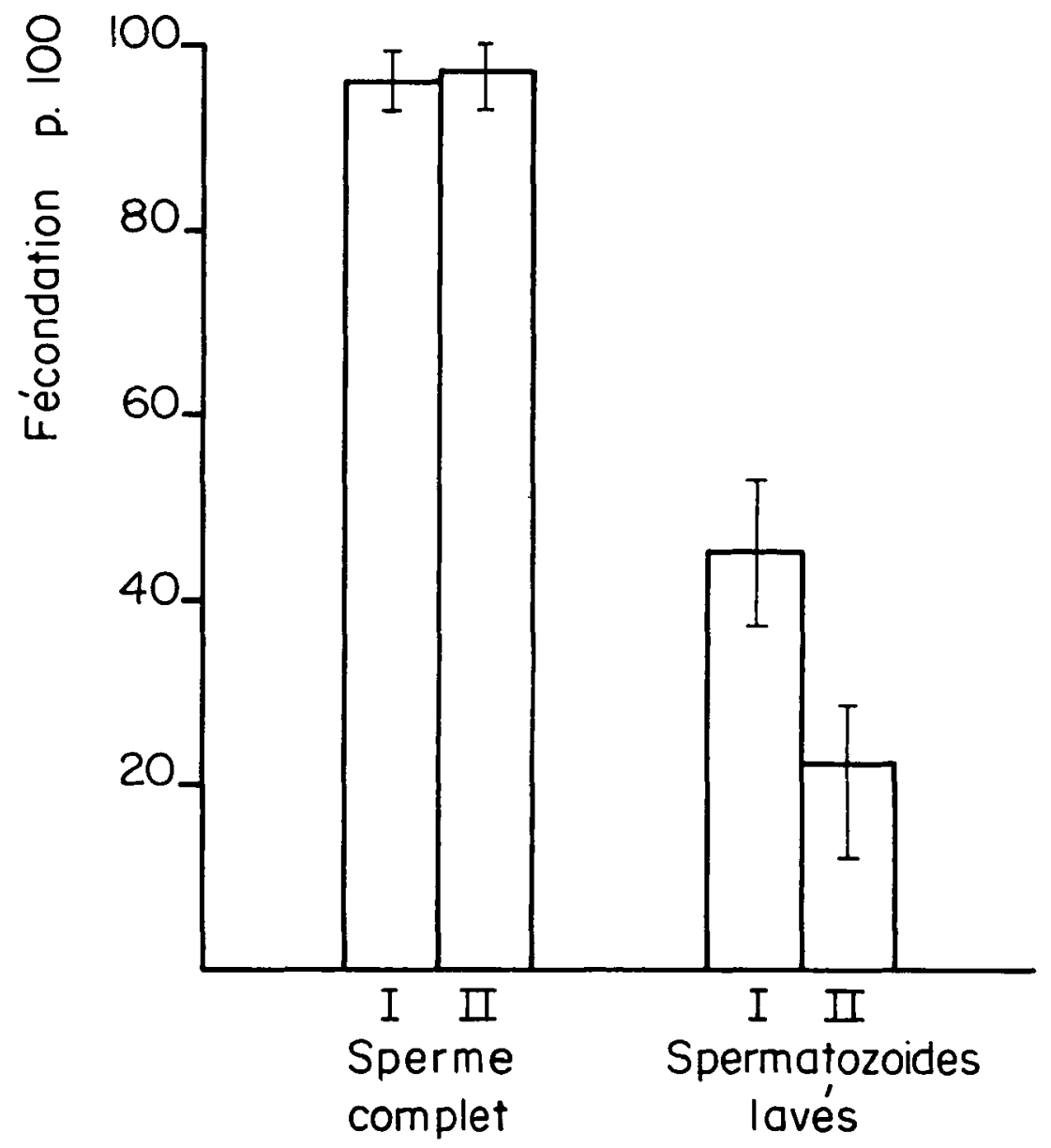

Figure 1: Effet du lavage des spermatozoídes sur le pourcentage de fécondation. L'insémination qui est réalisée avec le liquide colomique (I) et le dilueur d'insémination (II) porte sur du sperme complet (témoin), et des spermatozoïdes isolés puis lavés une minute dans 10 volumes de MMLS.

Dilutión finale du sperme: $1 / 1000$.

Concentration du sperme : $15,510^{-9}$ spermatozoïdes $/ \mathrm{ml}$.

Teneur en protéines : liquide ccelomique: $2,1 \mathrm{mg} / \mathrm{ml}$; liquide séminal : $1,7 \mathrm{mg} / \mathrm{ml}$. 
Deux protéines ont été utilisées: l'une dont les effets favorables ont déjà été démontrés chez les mammifères (HARRISON et al., 1978) Albumine sérique bovine, BSA (NBC grade V), l'autre pour un coût minime: l'ovalbumine (ovalbumin SIGMA grade II). Elles sont toutes deux incorporées au taux de $10 \mathrm{mg}$ par I dans DI et MMLS.

Le protocole expérimental consiste à exposer les ovules ou les spermatozoïdes aux deux milieux pendant des temps croissants et de procéder ensuite à l'insémination avec du sperme ou des ovules frais. Les lots d'ovules sont mélangés à $10 \mathrm{ml}$ de dilueur qui est éliminé et remplacé par DI lors de l'insémination.

Le sperme dilué au $1 / 1000(10 \mu l$ dans $10 \mathrm{ml}$ de DI) est versé sur des lots de 200 ovules 30,60, 90 et $150 \mathrm{sec}$. plus tard. Dans le cas d'exposition au MMLS, la technique de double dilution (BILLARD et JALABERT, 1974) est pratiquée lors de l'insémination le mélange MMLS + spermatozoïdes est versé sur des lots de 400 ovules environ préalablement additionnés de $10 \mathrm{ml}$ de DI. La concentration de $\mathrm{K}^{+}$est ainsi abaissée et les spermatozoïdes sont mis en mouvement.

Les mêmes gamètes sont utilisées dans les expériences 1 et 2 . Dans les autres cas, les ovules et spermatozoïdes proviennent de pools différents. Le nombre de spermatozoïdes par $\mathrm{ml}$ de sperme a été déterminé dans tous les cas sur cellule de Thoma. Les ovules sont prélevés par césarienne sur des femelles préalablement anesthésiées (Phénoxy-éthanol $0.5 \mathrm{ml} / \mathrm{l}$ ), saignées et essuyées afin d'éviter tout écrasement d'ovules et pollution du liquide cœlomique. Ce dernier est mis en pool et filtré sur membrane millipore $(1 \mu \mathrm{m})$ avant usage.

Les expériences se sont déroulées sous abri à température ambiante $10-12^{\circ} \mathrm{C}$. La concentration en protéines du liquide séminal et du liquide cœlomique ainsi que celle des milieux après insémination a été déterminée par la réaction de Folin.

La comparaison statistique des pourcentages de fécondation est faite par test $x^{2}$ après transformation angulaire des données. Sur les graphiques les pourcentages sont accompagnés de l'intervalle de confiance à 0,95 (Statistical tables. FISHER et YATES, 1963).

\section{RÉSULTATS}

\section{Expérience 1 - Effet du lavage des spermatozoïdes (fig. 1)}

Le lavage des spermatozoïdes de truite dans un milieu enrichi en $\mathrm{K}^{+}$où ils restent immobiles entraîne une diminution significative de la fécondance $(P<0.01)$ par rapport aux témoins (sperme complet). Cependant, lorsque l'insémination est pratiquée dans le liquide cœlomique, le pouvoir fécondant reste plus élevé qu'après insémination avec le DI (différence significative $P<0.05$ ).

\section{Expérience 2 - Effet du lavage des ovules (fig. 2)}

Le lavage des ovules dans des liquides physiologiques DIA ou MMLS entraîne une diminution significative de la fécondabilité $(P<0.01)$ qui $n$ 'apparaît que dans le cas de la plus forte dilution du sperme $\left(10^{-4}\right)$. Après lavage dans l'eau la chute de la fécondabilité est encore plus marquée.

\section{Expérience 3 - Effet du lavage combiné des ovules et spermatozoïdes (tabl. 1)}

Les résultats confirment les effets défavorables du lavage des spermatozoïdes ou des ovules et la combinaison des deux se traduit par une diminution encore accrue du taux de fécondation. L'analyse de variance portant sur la comparaison des traitements subis par les gamètes montre que le traitement des ovules provoque une diminution du pourcentage de fécondation plus marquée $(P<0.01)$ que le traitement du sperme $(P<$ 0.05).

Dans les trois expériences, la quantité de protéines dans le milieu après insémination n'est pas détectable $(<50 \mu \mathrm{g} / \mathrm{ml})$ dans tous les cas où les gamètes subissent un lavage, et à la limite de détection lorsque l'insémination est pratiquée avec DI.

\section{Expérience 4 - Effets de l'incorporation des protéines dans les dilueurs sur la survie des gamètes.}

La durée du pouvoir fécondant des spermatozoïdes dilués dans Dl est notablement prolongée dans le cas d'une supplémentation avec BSA mais pas avec l'ovalbumine (fig. 3). L'examen microscopique montre aussi que la motilité est prolongée après dilution dans DI'+ BSA. Lorsque le test porte sur la conservation des spermatozoïdes à l'état immobile, on constate que l'ovalbumine a un effet franchement défavorable (fig. 4); cela 


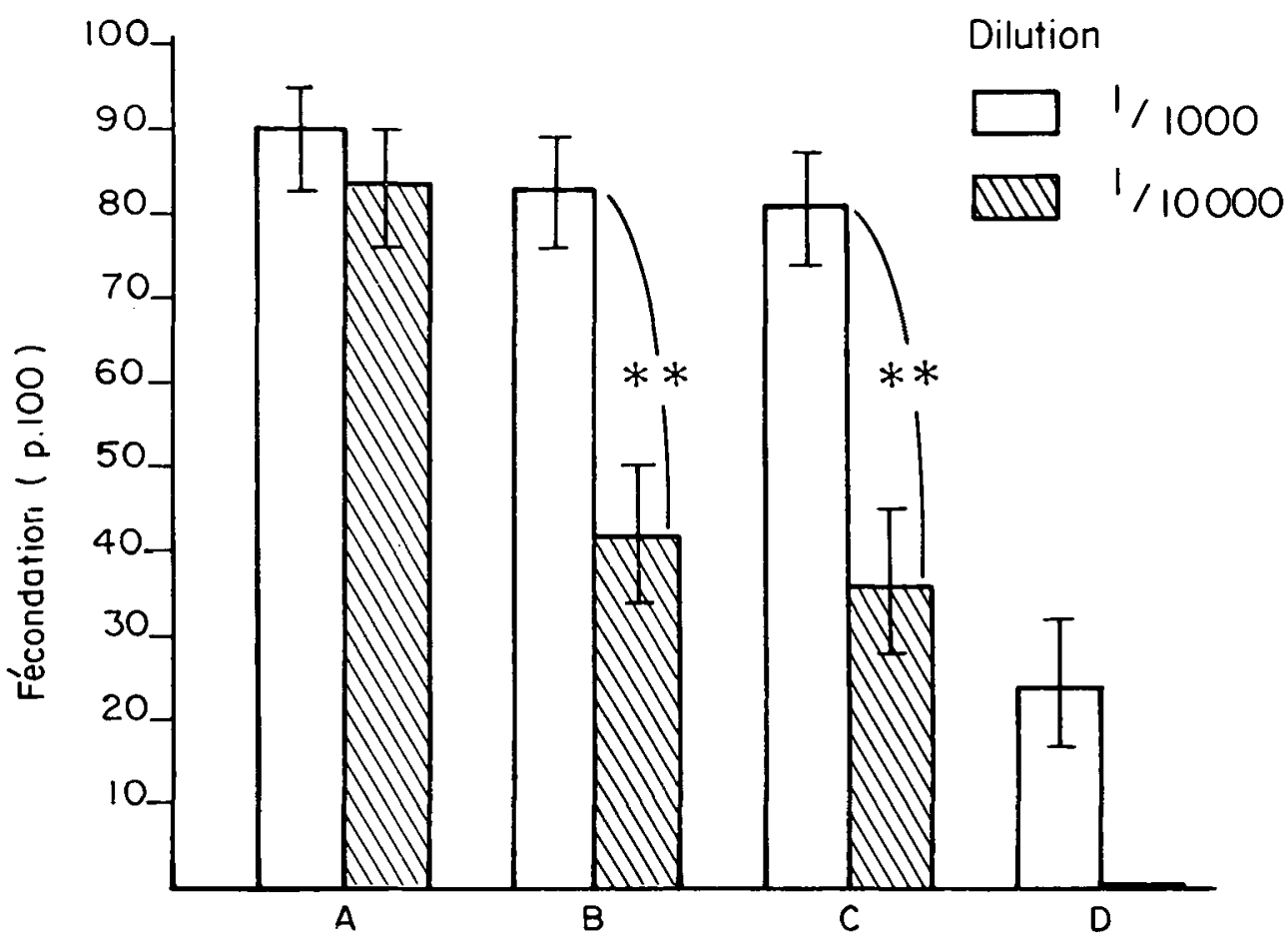

Figure 2 : Effets du lavage des ovules sur le pourcentage de fécondation. Après lavage de lots de 200 ovules environ dans $50 \mathrm{ml}$ de liquide coelomique (A), de dilueur d'insémination (DI) (B), de MMLS, (C) et d'eau douce (D), l'insémination par du sperme frais est pratiquée avec le dilueur de fécondation.

Dilution finale du sperme :

en blanc: $10^{-3}$

en hachuré : $10^{-4}$

Pourcentage de fécondation obtenu avec des ovules non lavés: 98,4 . $* *: \mathbf{P}<0.01$

\begin{tabular}{lccc}
\hline $\begin{array}{l}\text { Milieu de lavage } \\
\text { des ovules }\end{array}$ & $\begin{array}{c}\text { Milieu } \\
\text { d'insémination }\end{array}$ & $\begin{array}{c}\text { Sperme } \\
\text { complet }\end{array}$ & $\begin{array}{c}\text { Spermatozoïdes } \\
\text { lavés }\end{array}$ \\
\hline- & $\begin{array}{c}\text { liquide } \\
\text { cœlomique }\end{array}$ & $\begin{array}{c}68 \text { it } 12 \\
(1,5)\end{array}$ & $\begin{array}{c}52 \pm 11 \\
(1,6)\end{array}$ \\
\hline MMLC & DI & $\begin{array}{c}60 \pm 4 \\
\text { (ND) }\end{array}$ & $\begin{array}{c}39 \pm 4 \\
\text { (ND) }\end{array}$ \\
\hline DI & DI & $\begin{array}{c}29 \pm 6 \\
\text { (ND) }\end{array}$ & $\begin{array}{c}19 \pm 13 \\
\text { (ND) }\end{array}$ \\
\hline
\end{tabular}

Tableau 1 : Effet du lavage des gamètes sur le pourcentage de fécondation (dilution du sperme 1/1000) (concentration du sperme: 12,74 10'9 spermatozoïdes/ml).

Valeurs moyennes \pm écart type.

Les chiffres entre parenthèses correspondent aux teneurs protéiquẹs mesurées dans les milieux après insémination $(\mathbf{m g} / \mathbf{m l})$. ND = non détectable $(<50 \mu \mathrm{g} / \mathrm{ml})$. 

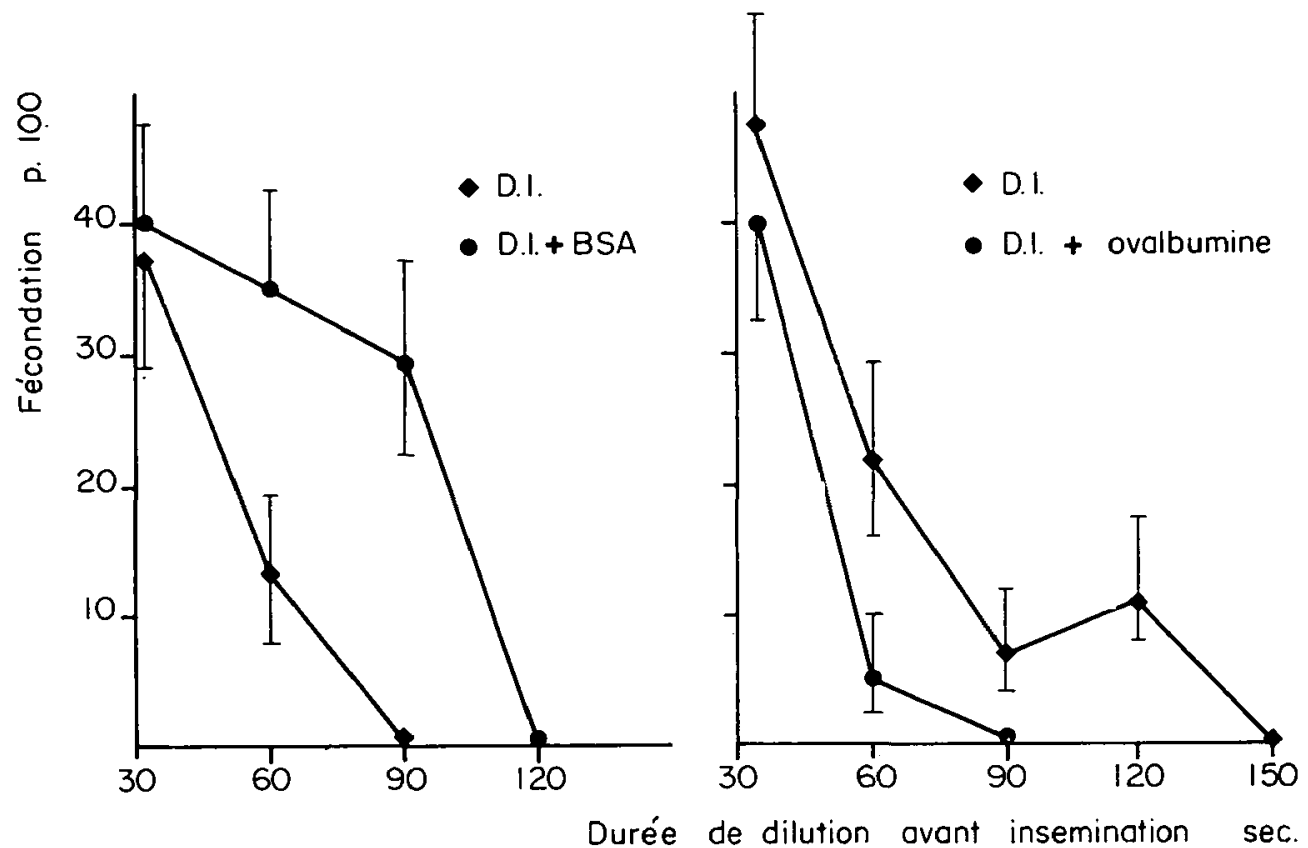

Figure 3 : Evolution du pouvoir fécondant du sperme après dilution au 1/1000 dans un milieu additionné ou non de BSA ou d'ovalbumine $(10 \mathrm{mg} / \mathrm{l})$

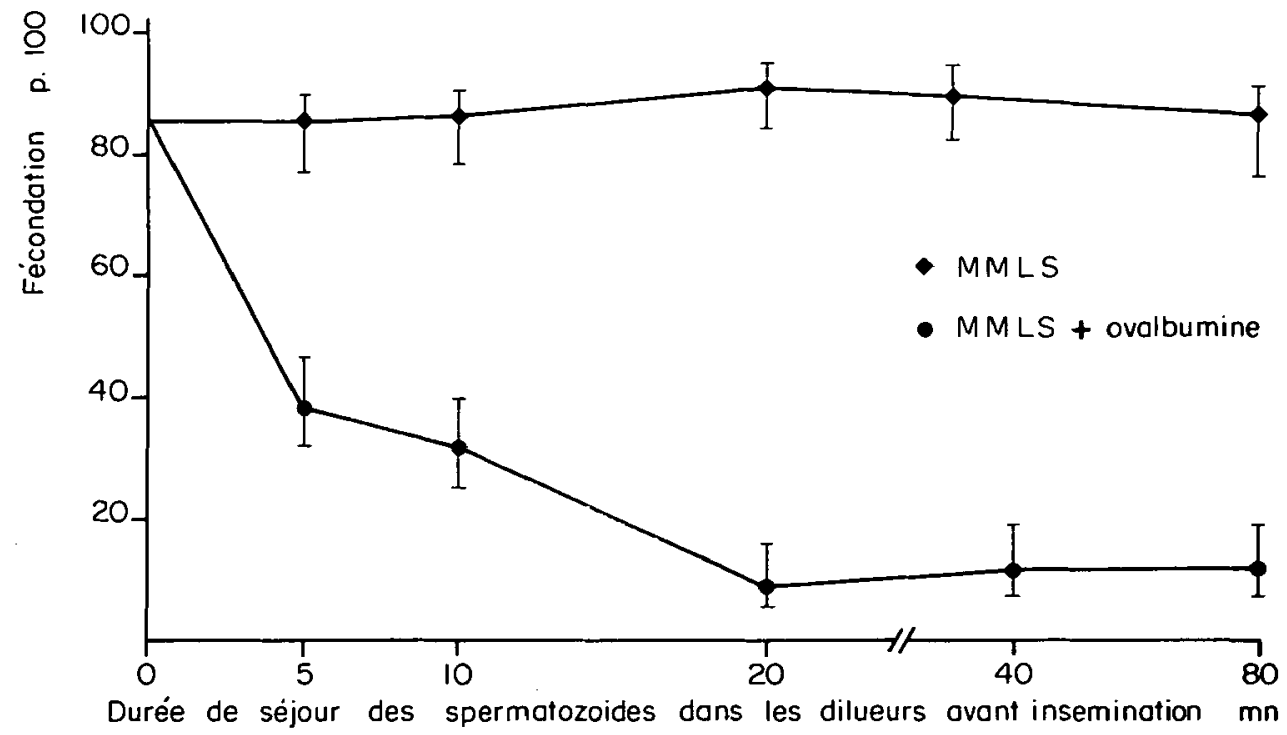

Figure 4 : Evolution du pouvoir fécondant du sperme dilué $(1 / 1000)$ et stocké pendant des temps croissants dans MMLS contenant ou non de l'ovalbumine.

est dû au fait que le MMLS contenant l'ovalbumine n'est pas éliminé et qu'après mélange avec le DI il se forme un précipité qui gêne les mouvements des spermatozoïdes. La présence de BSA ne modifie pas le pouvoir fécondant par rapport a MMLS au taux de dilution appliqué. La survie des ovules n'est pas significativement modifiée par la présence d'ovalbumine dans le DI (fig. 5 ). Il en est de même après incorporation de BSA; pourcentage de fécondation après 20 mn d'exposition : $38 \%$ dans DI contre $35 \%$ dans DI + BSA. Lorsque la BSA est incorporée à des taux croissants dans le DI, on n'observe pas de changement significatif dans le pourcentage de fécondation aussi bien à la dilution de $1 / 1000$ que $1 / 10.000$ (fig. 6). 


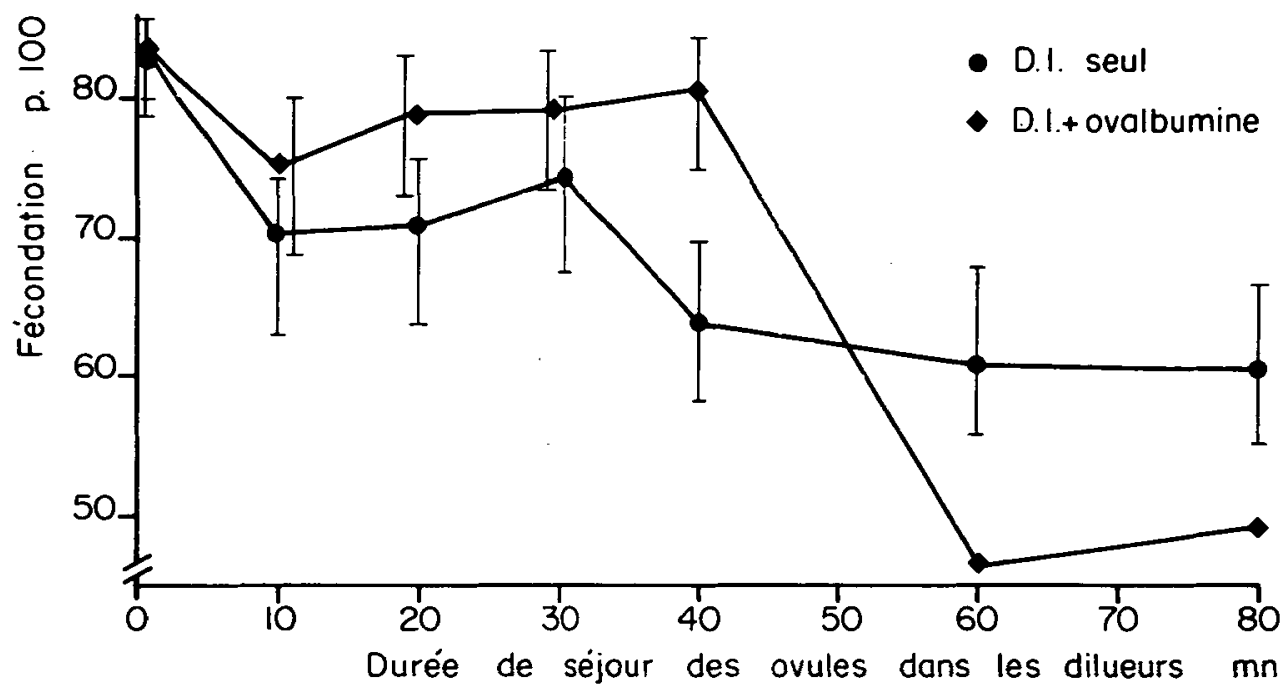

Figure 5 : Evolution de l'aptitude à la fécondation d'ovules dilués dans du DI additionné ou non d'ovalbumine.

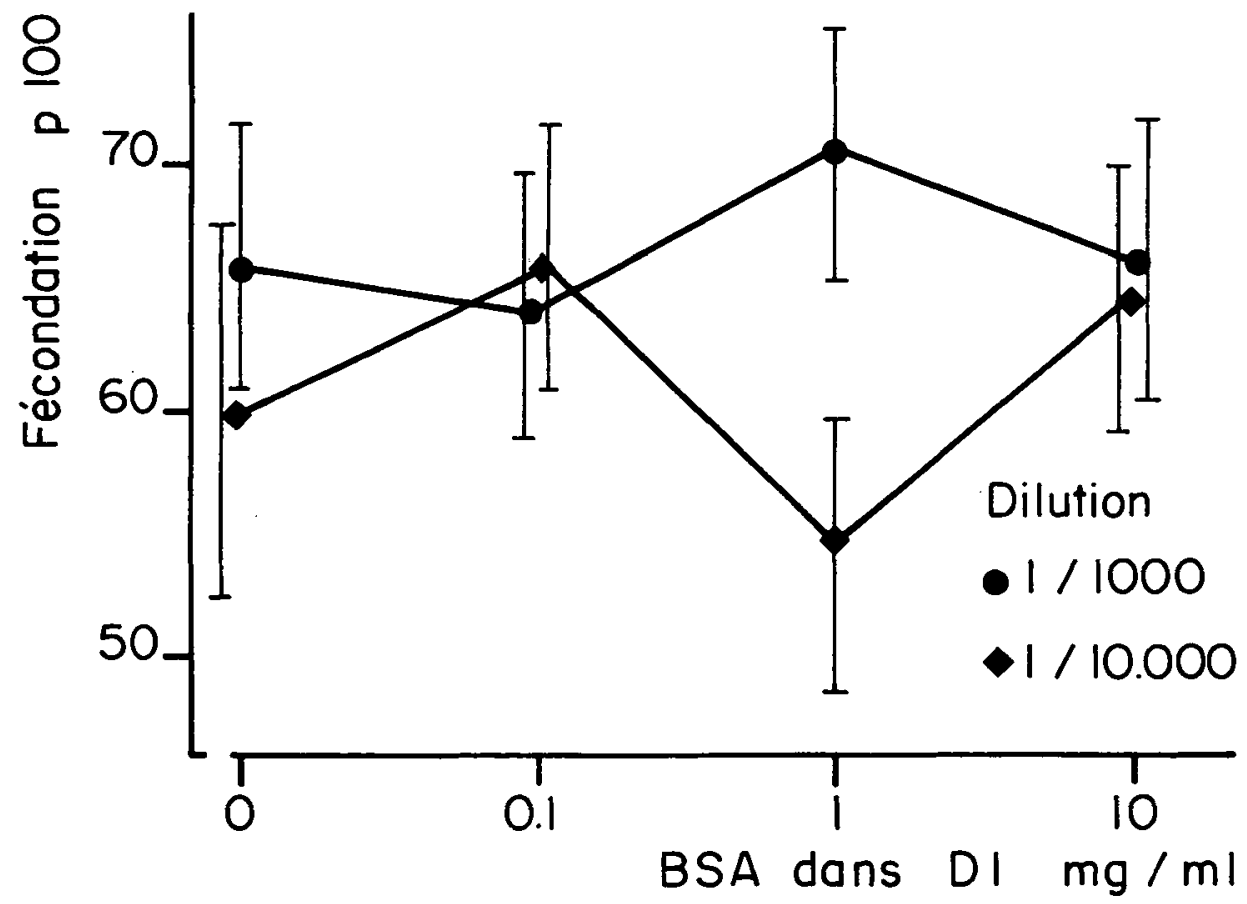

Figure 6: Pourcentage de fécondation obtenu après insémination d'ovules dans du DI contenant des quantités croissantes de BSA et pour deux taux de dilution différents.

\section{Discussion}

Le lavage des gamètes appliqué séparément à un seul d'entre eux ou aux deux diminue notablement leur fertilité, mais la simple dilution dans le DI telle qu'elle se pratique au cours des opérations d'insémination artificielle faisant appel à un dilueur (BILLARD, 1977 a) ne change pas le taux de fécondation en comparaison avec une insémination pratiquée dans le liquide cœlomique. Les essais de complémentation des dilueurs de conservation ou d'insémination par des protéines, pour compenser l'élimination du liquide cœlomique quelquefois indésirable, montrent que l'ovalbumine 
est le plus souvent défavorable et que seule la BSA à relativement forte dose $(10 \mathrm{mg} / \mathrm{ml})$ prolonge la durée de motilité et le pouvoir fécondant du sperme dilué. Cependant l'usage de BSA dans le DI utilisé pour l'insémination n'augmente pas le taux de fécondation même à la dilution de 1/10.000. Il apparaît donc inutile pratiquement d'enrichir en BSA le dilueur d'insémination commercial.

L'enlèvement du liquide séminal par centrifugation et lavage et son remplacement par un milieu inorganique (qui en restitue la composition minérale) a des effets néfastes sur le pouvoir fécondant. II se peut que les spermatozoïdes ainsi traités se trouvent dépourvus d'un substrat énergétique car le sperme est capable de métaboliser divers composants présents dans le liquide séminal (TERNER et KORSH, 1963 a-b; MOUNIB, 1967). Cependant, cette hypothèse est peu vraisemblable car la durée de motilité est brève et aucun apport exogène de composés divers, y compris C-AMP ou ATP, ne la prolonge notablement (BILLARD, non publié) à l'exception de la Théophylline (BILLARD, 1980). Le taux de dilution lors de l'insémination ou de la fécondation naturelle est tel que l'intervention de facteurs énergétiques originaires du liquide séminal est peu probable.

II est plus vraisemblable que les effets de la BSA soient dûs à une protection du spermatozoïde contre l'effet de dilution, comme cela a déjà été signalé chez les mammifères où les effets favorables de l'albumine sérique sur la survie des spermatozoïdes sont généralement interprétés comme résultant d'une protection contre le lavage (BREDDERMAN et FOOTE, 1971; HARRISON et al., 1978).

La chute de l'aptitude à la fécondation des ovules après lavage est compensée par une augmentation du nombre de spermatozoïdes mis en œuvre lors de l'insémination. II est alors possible que le lavage ait éliminé un facteur. favorisant la pénétration du spermatozoïde dans l'ovule. Il pourrait s'agir de substances de types gamone dont l'existence a été supposée chez les poissons par HARTMANN (1944) mais qui reste très hypothétique car non encore démontrée et même mise en question (TERNER et KORSH, 1963 b. BAYNES et al; 1981).

Il est aussi possible que le lavage éliminant toute trace de liquide colomique supprime le facteur de YOSHIDA et NOMURA (1972) stimulant la motilité du spermatozoïde. Le lavage peut aussi entraîner une élimination de tout facteur intervenant dans le métabolisme (MOUNIB et EISAN, 1969) ou provoquer une altération de la structure du micropyle comme le fait l'eau douce (SZÖLLÖSI et BILLARD, 1974), rendant plus difficile la pénétration du spermatozoïde. II existe une différence très importante entre une simple dilution (apport de $10 \mathrm{ml}$ de dilueur pour 200 ovules) qui ne provoque pas de perte de fécondabilité avant $40 \mathrm{mn}$ (BILLARD et JALABERT, 1974 ; BILLARD et al., 1974 b) (fig. 5) et un lavage dans $50 \mathrm{ml}$ de solution suivi d'un nouvel apport de $10 \mathrm{ml}$ lors de l'insémination, ce qui entraîne une perte importante de fécondabilité.

L'élimination d'éléments minéraux comme le $\mathrm{Ca}^{++}$'indispensable à la fécondation (GINSBURG, 1963; YAMAMOTO, 1961) n'est pas à exclure car l'emploi d'une simple solution de CINa (DI) pour l'insémination d'ovules lavés a des effets plus marqués que le MMLC qui contient du Cat+

En conclusion, si l'enlèvement complet des liquides biologiques qui accompagnent les gamètes altère leur fertilité, les ovules et les spermatozoïdes sont par contre en mesure de résister à des dilutions même élevées mais compatibles avec les techniques d'insémination faisant appel à un dilueur.

Cependant, ces résultats montrent que des précautions doivent être prises chaque fois que les gamètes seront dilués ou manipulés comme dans le cas de transport, de conservation à court terme ou de congélation. Les conditions de dilution devront alors faire l'objet d'attentions particulières; une supplémentation des milieux de dilution est alors à envisager et des investigations complémentaires sont nécessaires.

\section{REMERCIEMENTS}

Ce travail s'inscrit dans les relations contractuelles CSP-INRA. La maintenance des animaux a été assurée par MM. LEVILLAIN et BURGER. La présentation dactylographique du texte est due à $\mathrm{M}^{\mathrm{me}}$ CLAP.PIER C.

\section{REFERENCES BIBLIOGRAPHIQUES}

BAYNES S.M., SCOTT A.P., DAWSON A.P., 1981. Rainbow trout, Salmo gairdneri, spermatozoa: effects of cations and pH on motility. J. Fish Biol., 19, 259-267. 
BILLARD R., 1975 a. L'insémination artificielle de la truite Salmo gairdneri Richardson $V$ - Effets de la dilution et définition du rapport optimum gamètes/dilueur. Bull. Franç. Pisc. 257, 121-135.

BILLARD R., 1975 b. L'insémination artificielle de la truite Salmo gairdneri Richardson. IV - Effets des ions $\mathrm{K}^{+}$et $\mathrm{Na}^{+}$sur la conservation du pouvoir fécondant des gamètes. Bull. Franç. Pisc., 256, 88-100.

BILLARD R., 1977 a. A new techique of artificial insemination for salmonids using a sperm diluent. Fisheries, 1, 24-25.

BILLARD R., 1977 b. La production de truites arc-en-ciel au Japon. Pisc. Franc., 52, 20-28.

BILLARD R., 1977 c. Utilisation d'un système Tris-glycocolle pour tamponner le dilueur d'insémination de truite. Bull. Franc. Pisc., 264, 102-112.

BILLARD R., 1980. Prolongation de la durée de motilité et du pouvoir fécondant des spermatozoïdes de truite arc-en-ciel par addition de théophylline au milieu de dilution. C.R. Acad. Sci. Paris, 291, 649-652.

BILLARD R., JALABERT B., 1974. L'insémination artificielle de la truite (Salmo gairdner' Richardson). II - Comparaisons des effets de différents dilueurs sur la conservation de la fertilité des gamètes avant et après insémination. Ann. Biol. anim. Bioch. Biophys., 14, 601-610.

BILLARD R., PETIT J., JALABERT B., SZÖLLÖSI D., 1974 a. Artificial insemination in trout using a sperm diluant in J.M.S. BLAXTER. Early life history of fish, SpringerVerlag Heidelberg, 715-723.

BILLARD R., JALABERT B., BRETON B., 1974 b. L'insémination artificielle de la truite (Salmo gairdneri Richardson). III - Définition de la nature et de la molarité du tampon à employer avec les dilueurs d'insémination et de conservation. Ann. Biol. anim. Bioch. Biophys., 14, 611-621.

BREDDERMAN P.J., FOOTE R.H., 1971. Factors stabilizing bull sperm cell volume and prolonging motility at high dilution. Expt. Cell. Res., 66, 458-464.

DORIER A., 1949. Action du liquide cœlomique sur les spermatozoïdes de truite arc-enciel. Trav. Lab. Hydrobiol. Pisc., Univ. Grenoble, 41, 69-73.

FISHER R.A., YATES F., 1963. Statistical tables 6 th ed "Oliver and Boyd, EdinburghLondon edit.

GINSBURG A.S., 1963. Sperm-egg association and its relationship to the activation of the egg in salmonid Fishes. J. Embryol. exp. Morph., 11, 13-33.

GINSBURG A.S., 1968. Fertilization in fishes and the problem of polyspermy. Publishing house "Nauka", Moscou.

HARRISON R.A.P., DOTT H.M., FOSTER G.C., 1978. Effect of ionic strength, serum albumin and other macromolecules on the maintenance of motility and the surface of mammalian spermatozoa in a simple medium. J. Reprod. Fert., 52, 65-73.

HARTMANN M., 1944. Befruchtungsstoffe (Gamone) Ibei Fischen (Regenbogenforelle) Naturwiss., 32, 231.

LEITRITZ E., LEWIS R.C., 1976. Trout and salmon culture. Fish Bull. $n^{\circ}$ 107, State of California, Dept. of fish and game.

MANN T., 1956. Biochimie du sperme. P.U.F., $271 \mathrm{pp}$ :

MOUNIB M.S., 1967. Metabolism of pyruvate acetate and glyoxylate by fish sperm. Comp. Biochem. Physiol., 20, 987-992.

MOUNIB M.S., EISAN J.J., 1969. Metabolism of pyruvate and glyoxylate by eggs of salmon (Salmon salar). Comp. Biochem. Physiol., 29, 259-264.

NOMURA M., 1964. Studies on reproduction of rainbow trout, Salmo gairdneri, with special reference to egg taking - VI. The activities of spermatozoa in different diluents, and preservation of semen. Bull. Jap. Soc. Sci. Fish., 30, 723-733.

SZÖLLÖSI D., BILLARD R., 1974. The micropyle of trout eggs and its reaction to different incubation media. J. Microscopie, 21, 55-62.

TERNER C., KORSH G., 1963 a. The biosynthesis of fatty acid of glycerides and phosphatides by isolated spermatozoa of rainbow trout. J. Cell. Physiol., 62, 251 256.

TERNER C., KORSH G., 1963 b. The oxydative metabolism of pyruvate acetate and 
glucose in isolated fish spermatozoa. J. Cell. Comp. Physiol., 62, 234-250.

YAMAMOTO T., 1961. Physiology of fertilization in fish eggs. Inter. Rev. Cytol., 12, $361-405$.

YOSHIDA T., NOMURA M., 1972. A substance enhancing sperm motility in the ovarian fluid of rainbow trout. Bull. Jap. Soc. Sci. Fish, 38, 1073. 